\title{
Moderation-Mediation Effects in Bilingualism and Cognitive Reserve
}

\author{
Roberto R. Heredia ${ }^{1 *}$, Angélique M. Blackburn ${ }^{1}$ and Luis A. Vega ${ }^{2}$ \\ ${ }^{1}$ Department of Psychology and Communication, Texas A\&M International University, Laredo, TX, United States, \\ ${ }^{2}$ Department of Psychology, California State University-Bakersfield, Bakersfield, CA, United States
}

We first provide a critical review of the existing findings on bilingualism as a contributor to cognitive reserve from moderator-mediator warranting cause-effect research conclusions. We next address the question of direct or indirect effects between bilingualism and neurocognitive protective factors influencing the associated age-related mental deficits. The existing findings support bilingualism as a predictor and as a moderator. Third, we propose cognitive reserve models of bilingualism describing analytical approaches that allow testing of these models and hypotheses related to path strength and causal relationships between predictors, moderators, and mediators. Lastly and most importantly, we suggest using large datasets available via open repositories. This can aid in the testing of theoretical models, clarifying the roles of moderators and mediators, and assessing the research viability of multi-causal paths that can influence cognitive reserve. Creating collaborative datasets to test these models would greatly advance our field and identify critical variables in the study of the bilingual aging brain.

Keywords: bilingual cognitive reserve, bilingual moderators, bilingual mediators, cognitive reserve, mediating effects, moderating effects

\section{INTRODUCTION}

This paper is an attempt to clarify some issues that we see interchangeably used in the literature. These issues include mediators and moderators, each of which serves different purposes and has different assumptions and requirements that we clarify. A second purpose is to parse factors that are critical in the study of bilingual aging, specify the extent to which those factors amplify or diminish an effect (i.e., moderators), and determine whether a particular predictor (e.g., bilingualism) has an indirect effect on the outcome- or dependent-variable that is caused or mediated by a third variable. Identifying extraneous variables that moderate or mediate a causal relationship can clarify and advance our field. Third, we suggest other possible analytical approaches to data analyses that might be informative and allow for testing of models and specific hypotheses related to the path strength(s) between and among causal factors. Most importantly, we make a call for using large datasets related to this objective to help us increase understanding of bilingual cognitive research on aging using the Open Science Framework ${ }^{1}$ repositories. This can aid in the testing of theoretical models, clarifying the roles of moderators, and assessing the research viability of multi-causal paths that can influence cognitive reserve. Some data are already accessible through the open access philosophy of PLOS One, especially

${ }^{1}$ https://www.osf.io/ 
from those studies involving neurophysiological markers (fMRI and MRI) and others that are not readily accessible but might be in the future through Psychology's Study Preregistration run by The Center for Open Science. ${ }^{2}$ Creating collaborative datasets would greatly advance our field and identify critical variables in the study of the bilingual aging brain. We first review the general findings regarding the impact of bilingualism on cognitive reserve; second, we review how these important variables have been used, misused, or misinterpreted. Finally, we suggest plausible models that can be used as prototypes in model testing of theories of bilingualism and its influence/ relationship to cognitive reserve, through experimental or non-experimental data.

\section{COGNITIVE RESERVE}

As we age, neural networks begin to deteriorate and cognitive abilities decline. In the case of pathologies that impact a large percentage of elderly adults, such as Alzheimer's Disease (AD) and other forms of dementia, this decline has specific trajectories and consequences. However, some individuals experience a delay in exhibiting the initial symptoms of neural pathology and a slowed rate of its progression. Although their brains are already in early stages of deterioration, their cognitive abilities remain protected for a number of years. Socially, mentally, and physically engaging activities appear to mitigate the effects of cognitive decline (Fratiglioni et al., 2004). Of particular interest, mentally engaging activities such as formal education, mentally challenging occupations, and mentally stimulating leisure activities have been associated with resistance to the effects of neuropathology, in terms of expressing symptoms more slowly (e.g., Valenzuela and Sachdev, 2006a,b).

The association of cognitive resilience in the face of pathology and a history of mentally engaging activity has led to the cognitive reserve hypothesis (Valenzuela and Sachdev, 2006a,b) posing that complex mental (neural) activity throughout the lifetime protects against dementia by delaying the onset and rate of cognitive decline (Bialystok et al., 2007, p. 459; but see Van Dijk et al., 2008). Cognitive reserve is related to functional compensation for deterioration of neural pathways that may occur because some people perform cognitive tasks differently as a result of their experience. However, Woumans et al. (2015) point out that this compensation likely occurs through structural changes in the brain as a result of experience. These changes might include a combination of structural and functional factors such as increased resting phosphocreatine levels (Valenzuela and Sachdev, 2006b), better vascular support, enhanced neural conductivity, or compensation for deterioration in one pathway by using other neural pathways (Fratiglioni et al., 2004). Connections between neurons within a network can become strengthened as that network is repeatedly engaged, which modifies the proteins and enzymes along neural synapses. Neural connectivity can increase (or decrease) as the number of postsynaptic dopamine receptors in the mesocorticolimbic system

${ }^{2}$ https://www.cos.io change, neurotransmitter production is modified, synapses become enlarged, and/or dendritic spines grow on the postsynaptic neuron to increase the contribution of incoming information (Bliss et al., 2003; Nithianantharajah and Hannan, 2011; Freret et al., 2015; Blackburn, 2019; Iaccarino et al., 2020). Another possibility is that by engaging in tasks that require a high degree of cognitive effort, neural pathways for performing specific cognitive tasks are reorganized in a way that is more efficient and demands less resources (Bialystok et al., 2007; Woumans et al., 2015). More efficient networks may aid in retention of cognitive abilities when the networks first begin to deteriorate with age or pathology.

Factors associated with building cognitive reserve include social, mental, and physical engagements (Fratiglioni et al., 2004). However, many lifestyle habits associated with cognitive reserve involve frequent cognitive effort or mental exercise. Mentally demanding leisure activities, playing or listening to music, and playing video games have each been linked with increased reserve (e.g., Scarmeas et al., 2001; Bialystok, 2006; Basak et al., 2008). Formal education and occupational status are two of the most frequently identified predictors of preserving cognitive ability (e.g., Stern et al., 1994; Valenzuela and Sachdev, 2006b); however, some studies have shown that higher levels of education are linked to faster progression of AD (Scarmeas et al., 2006), and more demanding occupations are linked to earlier onset of $\mathrm{AD}$ symptoms (Woumans et al., 2015). It has been proposed that these factors may build cognitive reserve, but are associated with other risk factors for $\mathrm{AD}$, such as stress (Schweizer et al., 2012). Another explanation is that although high levels of cognitive reserve are linked to delayed onset of $\mathrm{AD}$, in some cases, decline is more rapid once the symptoms manifest (Stern et al., 1995, 1999). Finally, bilingualism, or the ability to speak two or more languages, is a skill that requires cognitive effort and interaction by many individuals on a regular basis. Similar to other mentally stimulating activities, bilingualism is linked to a delay in the onset and diagnosis of dementia (Bialystok et al., 2007), a robust finding that has been replicated multiple times (Anderson et al., 2020; Brini et al., 2020; Paulavicius et al., 2020). Although research on the relationship between the two is nascent, substantial and significant evidence has been found for bilingualism as a crucial factor that increases cognitive reserve.

\section{BILINGUAL COGNITIVE RESERVE}

Because bilingual advantage(s) later in life appear selective to the way in which bilinguals have used their languages, we can speculate that this added value is in fact a set of advantages that result from specific language skills that have been engaged across the lifespan. The bilingual advantage effect is the finding that bilinguals outperform monolinguals on tasks that require cognitive control - the ability to reactively and proactively respond to the demands of the environment (Paap et al., 2014). The cognitive advantages are rarely observed in young adults (for reviews and a meta-analysis, see Hilchey and Klein, 2011; Blackburn, 2017, 2018a, respectively), but are more often 
observed in older bilinguals. Even though behavioral advantages are not always found in young bilinguals, neural activity during cognitive control tasks is sometimes altered in young bilinguals compared to monolinguals (Kousaie and Phillips, 2012; Blackburn, 2013, 2018b). These neural effects in young adults are linked to enhanced behavioral performance in older adults (see also Morales et al., 2015; Kousaie and Phillips, 2017). Thus, as a result of managing their two or multiple languages throughout the lifespan, bilinguals develop cognitive reserve. One of the predominant explanations is that when a bilingual utters a word, the translation equivalent is thought to compete for selection, and interference from the competing language must be suppressed so that the appropriate word can be produced. The cognitive effort necessary to inhibit interference in order to remain in one language can develop neural networks involved in interference suppression, making this skill more efficient and resilient as the brain degrades with cognitive aging or pathology (Green, 1998; Bialystok et al., 2005, 2009).

The most convincing evidence of bilingual cognitive reserve comes from studies showing that bilinguals are slower to exhibit signs of dementia. This evidence was first reported by Bialystok et al. (2007) and was based on hospital records from dementia patients. Controlling for such variables as gender, education, and employment status, on average, bilinguals showed initial signs of dementia 4 years later than monolinguals, and they were diagnosed 3.2 years later ( 75.4 years of age for monolinguals vs. 78.6 years for bilinguals). A similar delay in the report of initial symptoms of 5.1 years for bilinguals was observed in a second study by the same research group of individuals diagnosed with AD (Craik et al., 2010).

Since these initial studies, researchers have replicated the results, finding delays in the onset and diagnosis of $\mathrm{AD}$, and other forms of dementia, even when controlling for potential confounds (for a review, see Freedman et al., 2014). Woumans et al. (2015) controlled for education, profession, and socioeconomic status (SES), and reported delays of 4.6 and 4.8 years for onset and diagnosis of $\mathrm{AD}$, respectively. Bialystok et al. (2014) controlled for diet, smoking, alcohol consumption, and physical and social activities. They found a 7.2 year-delay in the onset of $\mathrm{AD}$ and 3.5 year-delay in the onset of mild cognitive impairment (but no difference in the progression of the disease over the course of 1 year). These studies show consistent delays related to bilingualism in onset of symptoms and diagnosis of dementia.

Moreover, a more recent meta-analyses including eight studies found that on average, bilinguals exhibit $\mathrm{AD}$ symptoms 4.1 years and are diagnosed with $\mathrm{AD} 2.0$ years later than monolinguals (Paulavicius et al., 2020). Another confirmed a 4.7-year delay in $\mathrm{AD}$ onset and a 3.3-year delay in the diagnosis of dementia, but no delay in the diagnosis of mild cognitive impairment (Brini et al., 2020). An additional meta-analysis of 21 studies (18 of which provided the age of $\mathrm{AD}$ onset and six of which provided $\mathrm{AD}$ incidence) found that the delay in manifestation of $\mathrm{AD}$ symptoms has a moderate effect size, although the effect size of rate of $\mathrm{AD}$ incidence was weaker (Anderson et al., 2020). The overall conclusion from these studies was that the reported effects did not depend on education
(Brini et al., 2020), SES, or publication bias (Anderson et al., 2020). Together, these results suggest that bilingualism has a low likelihood of preventing dementia but it is likely to delay dementia by roughly 4 years in manifestation of $\mathrm{AD}$ symptoms. Most importantly, the meta-analysis demonstrated the need for high quality studies, which must observe requirements in conceptual-, methodological-, and statistical-rigor, while controlling for third variables such as culture and education (Grundy and Anderson, 2017; Woumans et al., 2017).

The above findings in bilinguals have been corroborated by comparisons of monolinguals and multilinguals showing less degeneration (Liu et al., 2012) and slower progression of dementia in multilinguals (Perani and Abutalebi, 2015). However, it should be noted that while bilinguals and multilinguals are often older than monolinguals at the time of onset and/or diagnosis, dementia has been shown to progress faster in bilinguals (Berkes et al., 2020). This is reminiscent of other factors, such as high levels of education, that are associated with rapid progression of decline after an initial delay of symptom onset (Stern et al., 1995, 1999). Thus, age and education appear to be moderating variables that need to be accounted for in the relationship between bilingualism and cognitive reserve.

\section{BILINGUAL AND SOCIAL FACTORS}

Bilingual studies have controlled for or specifically tested potential factors involved in cognitive reserve. These factors include the number of languages spoken, immigration status, country, age of second language acquisition, and factors known to impact cognitive reserve, such as education, occupational status, SES, premorbid intelligence, stimulating leisure activities, and mental, social, and physical activity (Guzmán-Vélez and Tranel, 2015).

Moreover, individual differences in the degree of bilingualism, such as early age of acquisition (Klein et al., 2014) and using multiple languages (e.g., Kavé et al., 2008; Chertkow et al., 2010; Perquin et al., 2013), appear to increase the likelihood of protection. Greater dementia delays are also sometimes observed for immigrant compared to non-immigrant populations (Chertkow et al., 2010; Mondini et al., 2014). But this effect may be due to the cognitive effort necessary to operate in a non-dominant language, as dominance in a language other than the L1 is associated with higher performance on cognitive tasks (Kavé et al., 2008). Frequently operating in a non-dominant language may also indicate greater proficiency or frequency of use, both of which are potential modulators of the bilingual advantage in cognitive reserve. It is equally possible that simply using two languages regularly enhances cognitive reserve, as dementia delays have been observed in non-immigrant populations regardless of whether the non-dominant (Alladi et al., 2013) or dominant language (Woumans et al., 2015) is spoken most frequently. In fact, the way in which bilinguals use their languages throughout their lives may engage cognitive control networks in different ways and increase cognitive reserve in the form of strengthening specific compensatory networks (for a review, see Blackburn, 2018a). Thus, individual language habits may play a large role in developing cognitive reserve. Importantly, bilingualism appears to 
interact with other factors known to enhance cognitive reserve, such as education as mentioned previously (cf. Kavé et al., 2008; Gollan et al., 2011; Hack et al., 2019).

\section{MODERATING AND MEDIATING EFFECTS OF BILINGUALISM}

So far, we have reviewed the evidence supporting bilingualism as an expression of cognitive reserve (see also, Bialystok et al., 2014; but see Zahodne et al., 2014; Mukadam et al., 2017a,b; Hack et al., 2019; Papageorgiou et al., 2019), in addition to other factors or predictors involved (e.g., education, IQ, occupation, SES, and immigration status). However, relationships between and among (predictors) factors are complex and might be modified by third variables (Fairchild and MacKinnon, 2009). In the experimental research domain, third variables such as confounds are held constant or statistically adjusted - provided these factors are quantitative, or treated as error variance through random assignment or counterbalancing measures. Other third variables include moderators, mediators, and suppressors. Suppressor variables can be identified when (a) the direct and indirect effects on the dependent variable are positive, negative, or vice versa (i.e., reciprocal suppressor); (b) when a predictor is added and receives a negative weight, and all other predictors are positively interrelated and yet the variance accounted for in the dependent variable increases (i.e., negative suppression); and (c) when by itself the predictor may have a very low correlation with the dependent variable but by virtue of a higher correlation with a second predictor, the original predictor's influence on the dependent variable increases (i.e., zero/classical suppressor).

Suppressor variables are sometimes called omitted variables (Bollen, 1989, p. 47-54), which are important to considered in structural equation models, where mediator and moderator relationships are examined and where the use of theory requires that all putative causal variables be included. Although we limit our discussion to moderators and mediators (Lancaster, 1999), the presence of omitted/suppressor variables is always lurking, requiring of the same rigor discussed in meta-analysis and requirements of high-quality studies. It is important that researchers have a solid foundation of theory in the bilingual memory field so that they can test the role(s) of suppressor variables that, if omitted or not anticipated, will bias their results. Briefly, a moderator (see Figure 1) qualifies a causal relationship as dependent on another variable in relation to its strength or direction, which we should note are also called direct effects. Of note, modulators or modulating effects, in the existing literature, are extensively included and are suggestive of moderators or moderating effects. It is not clear, however, if that is the case without delving into the operational definitions of the variables in question and how the analysis is carried out (see Kenny, 2019). Moreover, modulation effects are described even in the absence of interactive effects. A modulator, like a mediator must interact with the other independent variable (IV) or factor to be a true modulator. For example, Thorvaldsson et al. (2017) found that IQ moderated terminal decline in old age.
Specifically, higher IQs were associated with a delay in onset of terminal decline of up to 1.87 years on speed and 1.96 years on verbal ability. Additionally, Abutalebi et al. (2015) found that age moderated decrease of gray matter volume in the right dorsolateral prefrontal cortex for both bilinguals and monolinguals. An increase in age led to a decrease of gray matter. In the experimental literature, a moderation effect is typically an interactive effect, in which for example, results of an experimental treatment are qualified by gender, age, education, or any other theoretically related variable. In this way, the experimental manipulation is only effective for any variable that further limits, or clarifies, the causal relationship.

Mediators, or intervening effects (see Figure 2), in their simplest expression occur between the predictor and dependent variable in a causal fashion. Mediators account for the relationship between the predictor and dependent variable; the relationship between the predictor and dependent variable is not direct, but indirect via a third variable (Baron and Kenny, 1986; Fairchild and MacKinnon, 2009; Petrocelli et al., 2013; Kenny, 2019). Often mediators are hidden, or unknown, only to be identified through theory or previous work in the area. In the classic example, of whether smoking or vaping causes lung cancer, the mediating variable (which could be empirically measured) would be tissue damage. That is, the causal effect between smoking and lung cancer would be an indirect one. We should note that identifying or predicting moderators and mediators in bilingualism research has not been a standard practice.

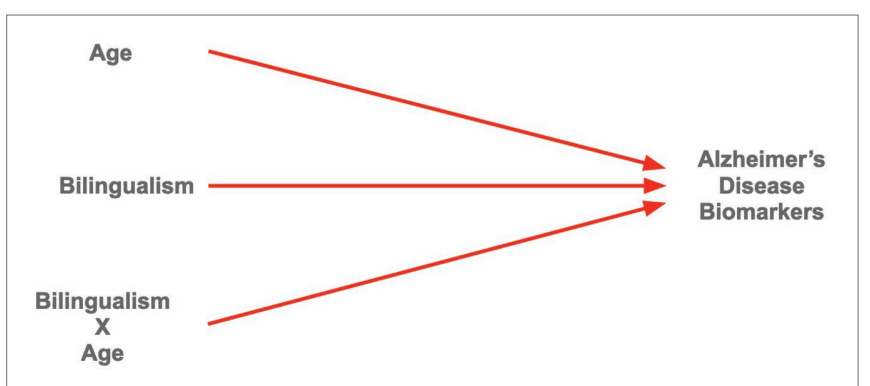

FIGURE 1 | Bilingualism as a moderator interacting with predictor age moderating Alzheimer's Disease (AD) markers (see Gollan et al., 2011).

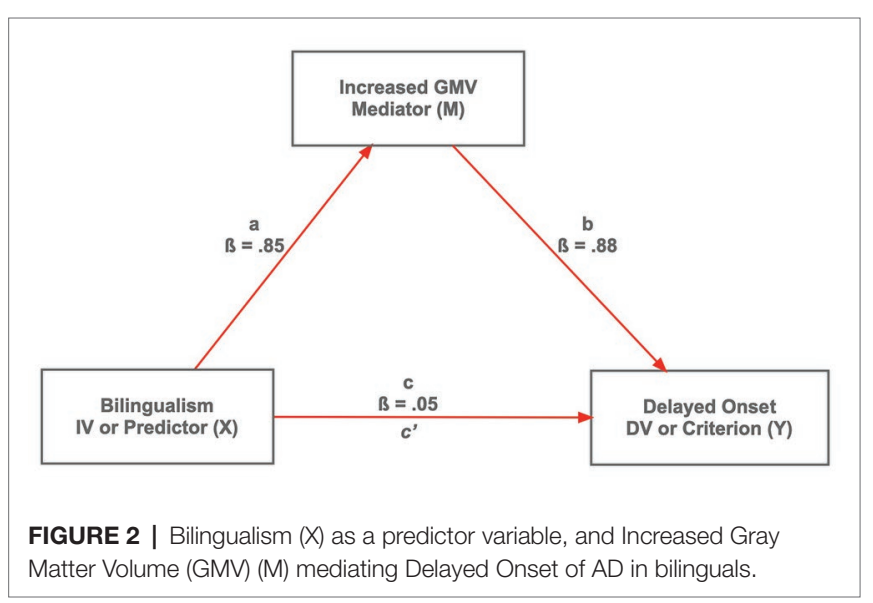


For example, in a search of 69 recent articles consulted for this paper, we found that mediating effects have been of little focus $(n=6)$, with greater emphasis on moderating $(n=8)$ or modulating $(n=18)$ effects, and with approximately $50 \%$ $(35 / 69)$ not giving any consideration to such effects.

\section{Moderator}

Bilingual research in this domain seeks to identify which variables (e.g., SES, IQ, education, and age) best predict cognitive outcomes associated with the aging brain. At issue is whether bilingualism (or multilingualism), controlling for such possible confounds as SES, IQ, and immigration status, protects against mental decline associated with old age. Regardless of the methodological approaches (experimental vs. correlational), the question of bilingualism and its protective cognitive qualities to aging could be posed in terms of causality or predictorbased analytical view assessing its contribution to the DV relative to other competing predictors or IVs. Of note, our operating assumptions are that methodological approaches (i.e., experimental vs. correlational), and analytical statistical techniques are orthogonal; binary responses (correct $=1$ and incorrect $=0$ ) that were typically converted into percentages and analyzed employing ANOVA can be best analyzed using generalized linear models or generalized mixed linear models (Jaeger, 2008). We first discuss bilingualism and putative causality in relation to moderators. This is important because moderators create dependency of the IV on the DV that needs to clarify if the causal relationship among the variables is confounded, spurious, or attenuated. We then review statistical paradigms that can best capture model testing in determining how bilingualism can be trusted as a strong predictor of cognitive reserve.

Is there a direct effect between bilingualism and mental faculties in the elderly? That is, what evidence exists or can be predicted of bilingualism as a moderator (see Figure 1) and/or a mediator (See Figure 2)? These questions need to be posed in framing hypothesis and a priori predictions in a research project if we expect internally valid results that can be generalizable across time, place, and people.

Figure 1 is an example of bilingualism as a moderator, interacting with the predictor age moderating levels of cerebral spinal fluid (CSF), which are AD biomarkers (Estanga et al., 2017). This interaction, as reported by Estanga et al. (2017) revealed that early bilinguals exhibited lower CSF levels as compared to late bilinguals and monolinguals, especially at older ages. Among other interesting effects moderated by bilingualism were lower prevalence of preclinical $\mathrm{AD}$ for early bilinguals. Although other studies discriminate between different levels of bilingualism and number of languages spoken, (e.g., Bialystok et al., 2007; Kavé et al., 2008; Alladi et al., 2013; Perquin et al., 2013; Bak et al., 2014; Zahodne et al., 2014; Woumans et al., 2015; Reyes et al., 2018; Hack et al., 2019), the study of Estanga et al. (2017) is unique in the sense that it is one of the few demonstrations that systematically conceptualizes bilingualism as a moderator, testing it, and clarifying that bilingualism influences CSF levels, which can have an effect on the aging brain. Gollan et al. (2011) provide an additional demonstration of education as a moderator, whereby years of education interacted with bilingualism, as measured by the Boston Naming Test, moderating age of Azheimer's Disease diagnosis; this moderating effect was driven by the bilinguals with low education. Other published studies also hint to the plausibility of bilingualism as a moderator. However, conclusions to the moderating effects of bilingualism are limited and inconclusive given methodological oversights considering the specific effects of bilingualism as a qualitative (i.e., levels or types), and quantitative (Gollan et al., 2011) and composite predictor variable (Calabria et al., 2020).

To illustrate, consider the above explanation and description of Figure 1, but including only bilinguals and monolinguals; the bilingual type (early vs. late) would not be central to the hypothesis at hand. Most likely, the interaction between age and bilingualism would show bilinguals with lower CSF levels than monolinguals, especially at advanced age levels (60 years and above). One possible interpretation, and a prevalent one by some scholars, of a significant interaction might be that, lower CSF AD biomarker levels are moderated by bilingualism, as we make the additional assumption that learning other language(s) has been a long learning experience. However, it is important to note that language group (bilingual vs. monolingual or single vs. dual language), in this particular case, would be a plausible moderator but not bilingualism, though the intuition would be probably in the right direction (see Thorvaldsson et al., 2017; Kraemer et al., 2019, for excellent examples of moderation analyses). One possible objection to our argument is that levels of bilingualism as a factor could operationally be defined as bilingual vs. nonbilingual, where the monolingual group is actually a level of the variable. [It would be correct, however, if the focal factor's levels involve number of languages spoken (e.g., monolingual, bilingual, trilingual, etc.)]. This would be, without any doubt, a classic case of construct validity, and one would have to rely on the extensive literature on bilingualism and the various ways in which bilingualism can be classified or conceptualized (e.g., simultaneous, early, late, compound, coordinate, sequential, successive, low, high proficiency, beginner, and advanced; Heredia and Cieślicka, 2014; see also Gollan et al., 2011; Calabria et al., 2020). Clearly, this research domain would benefit from studies specifically looking at the moderating effects of bilingualism on the aging brain. Excellent literature reviews of the effects of bilingualism and cognitive reserve are provided by Calvo et al. (2016) and Klimova et al. (2017), and recent meta-analyses by Mukadam et al. (2017b); Anderson et al. (2020); Brini et al. (2020); Paulavicius et al. (2020). None of these articles make reference to the plausibility of bilingualism as a moderator, or equally if not more important, as a mediator, to which we now turn.

\section{Mediator}

What is the relationship between bilingualism and neuro-cognitive mental decline associated with old age? Is there a direct (causal) relationship between bilingualism and neurocognitive protective effects such as diet, education, intelligence, or other internal agents that in turn influence (mediating causal effects) associated with the age-related mental deficits of the aging bilingual? A review of the existing bilingual aging literature point to an indirect 
effect as summarized by Figure 2 [e.g., Luk et al., 2011; Schweizer et al., 2012; Perquin et al., 2013; Abutalebi et al., 2014, 2015; Perani et al., 2017; Reyes et al., 2018; cf. Rossi et al., 2017; cf. Kavé et al., 2008; Mukadam et al., 2017a,b; and see Grady et al., 2015, for a similar proposed mediation model with lifelong use of two languages as predictor, and stronger (neuro) functional connections and greater (neuro) modulation of task-related activity mediating executive control].

Figure 2 describes a mediation model with two causal paths: The direct link, path $c$, between Bilingualism as predictor (X) and Delayed Onset of Alzeimer's Disease as criterion $(\mathrm{Y})$; direct link, path $b$, between Increased GMV (M) and Y. There is also path $a$, the possibility to which most of the evidence summarized points (see Grady et al., 2015, for a similar argument). Figure 2 provides a simple and straightforward testable model; it would be possible, indeed, to include possible moderators (e.g., education and SES) and mediators (e.g., executive control; see Petrocelli et al., 2013, for a discussion of complex moderation-mediation models; cf. Grady et al., 2015). Whether bilingualism has a direct (path c) or indirect effect (paths $a+b$ ) on Delayed Onset of Alzheimer's or Mental Agility remains to be empirically verified. Utilizing the appropriate regression-based analytical techniques (e.g., path analysis or structural equation modeling), the hypotheses is that path a and $b$ would reveal large unstandardized weights $(B s)$ or standardized regression coefficients ( $\beta s$; see Figure 2 ), as compared to a nonsignificant Path $c$ after controlling for $M$ could be tested (e.g., Preacher and Hayes, 2008; Fairchild and MacKinnon, 2009; Yzerbyt et al., 2018; see Sauter et al., 2019, for an excellent demonstration of a mediation analysis related to aging and cognitive reserve). Methodological paradigms do not and should not determine the statistical approach. ANOVA has been crucial in experimental psychology; however, other regression-based-statistical techniques (e.g., linear mixed models and generalized mixed models) are crucial in model testing (e.g., Ljungberg et al., 2013; Perquin et al., 2013; Zahodne et al., 2014; Klein et al., 2016; Hack et al., 2019) and overcoming limitations of traditional regression analysis in dealing with repeated measures designs (Lorch and Myers, 1990). Indeed, these statistical techniques required more attention (cf. Bak et al., 2014; Xie and Zhou, 2020).

The existing literature, thus far, supports bilingualism as a predictor and as a moderator. We now consider the viability of bilingualism, as measured in a continuum (Gollan et al., 2011; see also Calabria et al., 2020) as a plausible mediator. Mediators, as argued by Baron and Kenny (1986) explain how external physical events take on internal psychological significance (p. 1176). So, a mediator could conceivably be viewed as a mechanism that might explain why bilinguals, for example, consistently outperform monolinguals on attentional tasks tapping into executive control (but see Paap, 2018). At a very general level, it might come across as a reasonable explanation. However, from a moderator-mediator perspective, for a mediator status, the causal effects of bilingualism can be predicted from other predictors or markers. Possible predictors of bilingualism or multilingualism can include positive attitudes toward multiculturalism and diversity, language-group/ethnicity as positively viewed, language-group/ethnicity as politically significant, levels of education, SES and immigration status (see for example Simonton, 2008). Additionally, bilingualism could be a conglomeration or a multidimensional composite of multiple predictors and moderators interacting (see for example, Calabria et al., 2020). Figure 3 provides such possibility. The model described in the figure suggests a structure in which immigration as a predictor interacts with education, the moderator, to predict bilingualism as a mediator. In turn, bilingualism develops a causal path $\left(b_{1}\right)$ to the Executive Control, the dependent variable.

If bilingualism is a mediator, a prediction would be that paths $a_{3}$ and $b_{1}$ would reveal large and significant $\beta s$ relative to $a_{1}-a_{2}$, with nonsignificant paths for $c_{1}^{\prime}-c_{3}^{\prime}$ (see discussion above) and the potential moderating effects of the three-and two-way interactions described by paths $j, h$, and $b_{2}$. Noticed that the model in Figure 3 could be modified further to incorporate working memory capacity. If we are to assume that bilinguals have larger working memory capacity, a correlate of cognitive reserve, it can be assumed that high working memory capacity in bilinguals moderates executive control. However, Martin et al. (2020) found that working memory capacity (path $e_{1}$ ) was not significant once general intelligence was included into the model. That is, they found no moderation effect, but rather that the role of working memory capacity on the effect of learning (i.e., cognitive reserve) was strictly mediated through intelligence (paths $d_{3}$ and $e_{2}$ ). This is but a small glimpse on the power of using large datasets and including moderating and mediating effects in models of bilingualism and the aging brain. These proposed models, could very well be modified, and improved even further, and tested using newly developed procedures greatly simplified by existing open source statistical program (e.g., med-mod procedure of jamovi.org). To our knowledge, no study, correlational or otherwise, has addressed this possibility employing such statistical techniques as confirmatory factor analysis or structural equation modeling. Existing studies with large datasets (Kavé et al., 2008; Alladi et al., 2013, 2016; Ljungberg et al., 2013; Bak et al., 2014; Zahodne et al., 2014; Mukadam et al., 2017a; Hack et al., 2019) if made accessible via the Open Science Network would be a positive step in developing and testing bilingual models of bilingual cognitive reserve.

\section{MODERATION-MEDIATION ANALYTICAL ISSUES}

To systematically identify other possible third variables, moderating or mediating bilingual cognitive reserve effects and the bilingual aging process, additional multivariate studies (i.e., correlational) of the type reported by Kavé et al. (2008); Ljungberg et al. (2013); Bak et al. (2014); Zahodne et al. (2014); Alladi et al. (2016); Klein et al. (2016); Mukadam et al. (2017b); Burke et al. (2019); and Hack et al. (2019) are crucial for model testing and advancing the field. As it has been argued (e.g., Cronbach, 1975), variance of third variables such as moderators and mediators go often unaccounted for. Typically, 


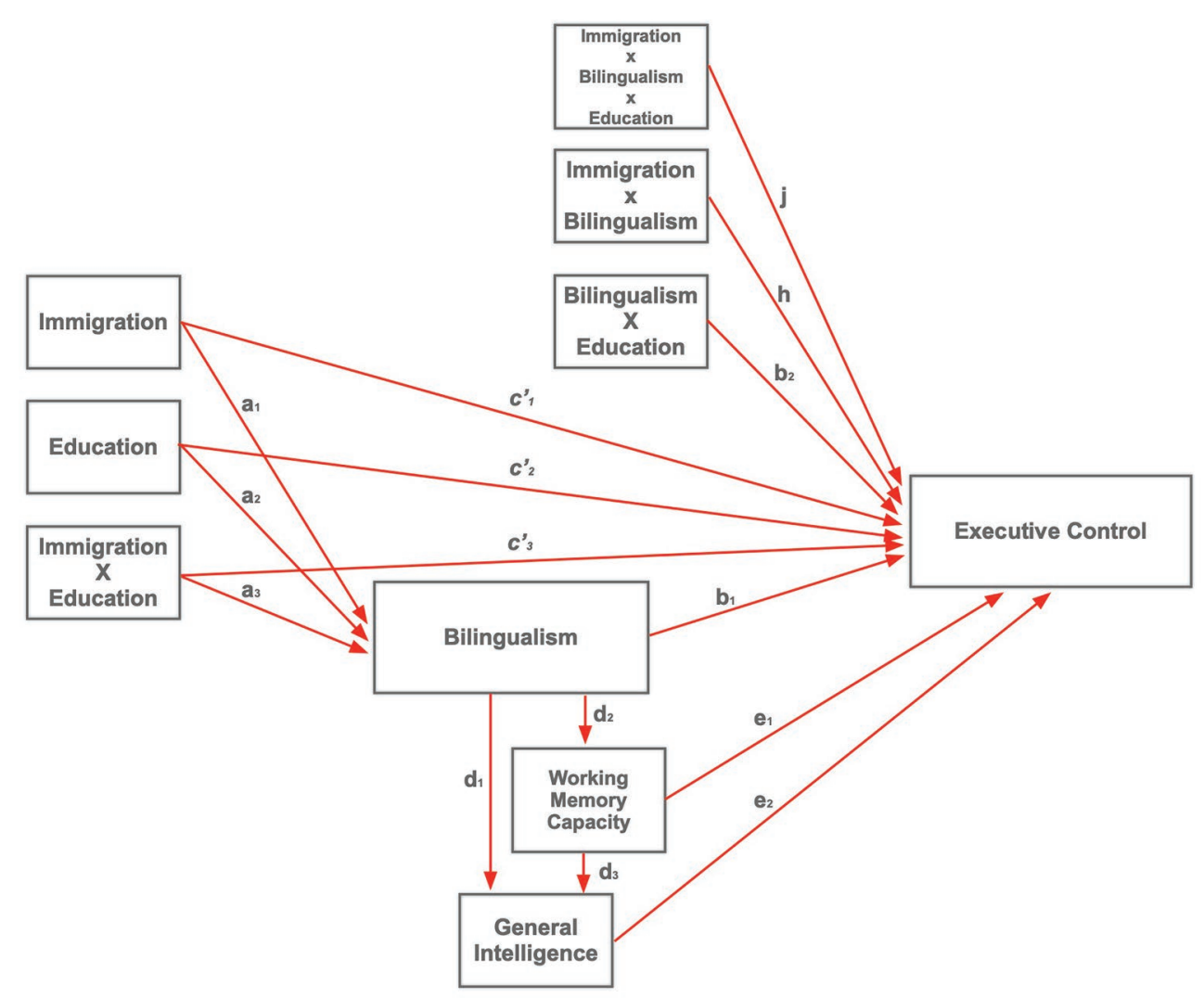

FIGURE 3 | A hypothetical mediation (bilingualism) and moderation (immigration, education, immigration $\times$ education; immigration $\times$ bilingualism $\times$ education, immigration $\times$ bilingualism, and bilingualism $\times$ education) model of bilingual cognitive reserve (cf. Fairchild and MacKinnon, 2009); working memory capacity (attention span), and general intelligence (cf. Martin et al., 2020).

these factors are not identified, measured, or analyzed appropriately. While it is true that theoretically important variables in an experiment, for example, can be treated as covariates, this can only be true if the factor is a quantitative one (e.g., Gollan et al., 2011). Many third variables relevant to bilingualism may not meet that standard such as monolingual versus bilingual, immigrant versus non-immigrant, or even the ubiquitous variable of gender. The use of hierarchical regression, as in linear mixed modeling approaches, in particular which theoretically considers how variables should be entered into the regression equation, would allow us to estimate variance value added by each variable. With this logic, it can be seen that a moderator is simply the interaction entered into the regression equation, with significance testing to assess for variance added over and beyond the DV or main predictor, in addition to other indexes such as effect size for the interaction (see moderator discussion section). Test of a moderator in regression is also said to be a test of the direct effect of that variable on the dependent variable (path $c$ in Figure 2; and all paths in Figure 3 with the exceptions of $a_{1-3}$ and $d_{1-3}$ ). In turn, a mediator is said to be an indirect effect. As stated earlier, the influence of a predictor is mediated through a theoretically important third variable, such that the total effect on the dependent variable is the product of the predictor times the third variable (product of paths $a$ and $b$ in Figure 2; paths $a_{1-3}$ and $d_{1-3}$ in Figure 3). Although our previous discussion placed multiple regression at the discussion of moderators and mediators, the important aspect for the current discussion is that non-experimental approaches can be useful in assessing the effects of third variables.

Furthermore, to better understand the role of moderators (direct effects) and mediators (indirect effects), latent variable models can be constructed and tested. Such approach uses regression as a foundation, but only with multiple structural equations that allow for model testing using best fit indexes, and model comparisons (see discussion above). Again, as in hierarchical regression, theory and a priori predictions are required to test these models, which can be said are a reflection of reality, and considers the weight or influence of all variables (e.g., predictors, third variables, exogenous, or endogenous). In a manner similar to confirmatory factor analysis, the models tested can involve the measurement model (observable variables such as education, bilingual type, and learning contextual modes) or the latent, or construct model (unobserved variables such as cognitive reserve). While the computational mathematics behind such models is complex, interested readers are directed to Kenny (2019) for an excellent exposition of these techniques. In a nutshell, structural equation modeling 
involves regression, factor analysis, and often the maximum likelihood estimation of fitting functions. For instance, a chi-square statistic is used to accept (nonsignificant) or reject (significant) the proposed model, in addition to several indexes that assess fit (closer to 1.0, the better). As stated, open source statistical programs (e.g., jamovi.org and jasp-stats. org) have significantly simplified the analytical process without having to learn the complicated computations. With most new statistical programs being menu driven, it is nonetheless critical that researchers planning to use structural equation modeling have basic, practical knowledge of what those statistical tools entail. We recommend Kenny (2019) for an assortment of sources for mastery of these revolutionary statistical techniques.

\section{CONCLUSION}

Bilingualism is just one of many factors thought to protect against age- and pathology-related cognitive decline. Bilingualism is linked to some advantages in cognitive performance and delays in dementia onset and diagnosis. We have reviewed the literature and suggested other possible analytical approaches to data analyses that might be more informative and which allow for testing of models and specific hypotheses related to the path strength(s) between and among causal factors. Most importantly, we suggest using large datasets using the Open Science Framework (see text footnote 1) repositories.

\section{REFERENCES}

Abutalebi, J., Canini, M., Della Rosa, P. A., Sheung, L. P., Green, D. W., and Weekes, B. S. (2014). Bilingualism protects anterior temporal lobe integrity in aging. Neurobiol. Aging 35, 2126-2133. doi: 10.1016/j.neurobiolaging.2014.03.010

Abutalebi, J., Guidi, L., Borsa, V., Canini, M., Della Rosa, P. A., Parris, B. A., et al. (2015). Bilingualism provides a neural reserve for aging populations. Neuropsychologia 69, 201-210. doi: 10.1016/j.neuropsychologia.2015.01.040

Alladi, S., Bak, T. H., Duggirala, V., Surampudi, B., Shailaja, M., Shukla, A. K., et al. (2013). Bilingualism delays age at onset of dementia, independent of education and immigration status. Neurology 81, 1938-1944. doi: 10.1212/01. wnl.0000436620.33155.a4

Alladi, S., Bak, T. H., Mekala, S., Rajan, A., Chaudhuri, J. R., Mioshi, E., et al. (2016). Impact of bilingualism on cognitive outcome after stroke. Stroke 47, 258-261. doi: 10.1161/STROKEAHA.115.010418

Anderson, J. A., Hawrylewicz, K., and Grundy, J. G. (2020). Does bilingualism protect against dementia? A meta-analysis. Psychon. Bull. Rev. doi: 10.3758/ s13423-020-01736-5 [Epub ahead of print]

Bak, T. H., Nissan, J. J., Allerhand, M. M., and Deary, I. J. (2014). Does bilingualism influence cognitive aging? Ann. Neurol. 75, 959-963. doi: 10.1002/ana.24158

Baron, R. M., and Kenny, D. A. (1986). The moderator-mediator variable distinction in social psychological research: conceptual, strategic, and statistical considerations. J. Pers. Soc. Psychol. 51, 1173-1182. doi: 10.1037//0022-3514.51.6.1173

Basak, C., Boot, W. R., Voss, M. W., and Kramer, A. F. (2008). Can training in a real-time strategy video game attenuate cognitive decline in older adults? Psychol. Aging 23, 765-777. doi: 10.1037/a0013494

Berkes, M., Bialystok, E., Craik, F., Troyer, A., and Freedman, M. (2020). Conversion of mild cognitive impairment to Alzheimer Disease in monolingual and bilingual patients. Alzheimer Dis. Assoc. Disord. 34, 225-230. doi: 10.1097/ WAD.0000000000000373

Bialystok, E. (2006). Effect of bilingualism and computer video game experience on the Simon task. Can. J. Exp. Psychol. 60, 68-79. doi: 10.1037/cjep2006008
This can aid in the testing of theoretical models, clarifying the roles of mediators and moderators, and assessing the research viability of multi-causal paths that can influence cognitive reserve. One potential avenue for data sharing is Psychology's Study Preregistration run by The Center for Open Science (see text footnote 2). Creating collaborative datasets would greatly advance our field and identify critical variables in the study of the bilingual, aging brain.

\section{AUTHOR CONTRIBUTIONS}

$\mathrm{RH}, \mathrm{AB}$, and LV contributed equally in the development and final product of the present manuscript. All authors contributed to the article and approved the submitted version.

\section{FUNDING}

Preparation of this manuscript was made possible by the US Department of Education, TAMIU Advancing Research and Curriculum Initiative Grant P031S190304.

\section{ACKNOWLEDGMENTS}

We thank Anna B. Ciéslicka and Tanja Angelovska for excellent comments and suggestions on earlier drafts.

Bialystok, E., Craik, F., Binns, M., Ossher, L., and Freedman, M. (2014). Effects of bilingualism on the age of onset and progression of MCI and AD: evidence from executive function tests. Neuropsychology 28, 290-304. doi: 10.1037/neu0000023

Bialystok, E., Craik, F. I., and Freedman, M. (2007). Bilingualism as a protection against the onset of symptoms of dementia. Neuropsychologia 45, 459-464. doi: 10.1016/j.neuropsychologia.2006.10.009

Bialystok, E., Craik, F. I., Green, D. W., and Gollan, T. H. (2009). Bilingual minds. Psychol. Sci. Public Interest 10, 89-129. doi: 10.1177/1529100610387084

Bialystok, E., Martin, M. M., and Viswanathan, M. (2005). Bilingualism across the lifespan: the rise and fall of inhibitory control. Int. J. Biling. 9, 103-119. doi: 10.1177/13670069050090010701

Blackburn, A. M. (2013). A study of the relationship between code switching and the bilingual advantage: Evidence that language use modulates neural indices of language processing and cognitive control. (Ph.D. Neurobiology). San Antonio: University of Texas.

Blackburn, A. M. (2017). “The Bilingual brain” in An introduction to Bblingualism: Principles and processes. 2nd Edn. eds. J. Altarriba and R. R. Heredia. (New York: Routledge), 107-138.

Blackburn, A. M. (2018a). "Cognitive impact of bilingualism and language habits at the borders of cultures and nations" in Inquiries into literacy learning and intercultural competency in a world of border tensions. eds. T. Huber and P. S. Roberson (Charlotte, NC: Information Age Publishing), $141-166$

Blackburn, A. M. (2018b). "Defining, characterizing, and recruiting participants according to a novel distinction for an event-related potential study regarding the cognitive impact of code-switching habits" in SAGE research methods cases in psychology.

Blackburn, A. M. (2019). "Neuroimaging studies of multilingual speech" in The handbook of the neuroscience of multilingualism. eds. J. W. Schwieter and M. Paradis (Hoboken, NJ: John Wiley \& Sons), 121-146.

Bliss, T. V. P., Collingridge, G. L., Morris, R. G. M., Harris, K. M., Fiala, J. C., and Ostroff, L. (2003). Structural changes at dendritic spine synapses during 
long-term potentiation. Philos. Trans. R. Soc. Lond. B Biol. Sci. 358, 745-748. doi: $10.1098 /$ rstb.2002.1254.

Bollen, K. A. (1989). Structural equations with latent variables. New York: John Wiley \& Sons.

Brini, S., Sohrabi, H. R., Hebert, J. J., Forrest, M. R., Laine, M., Hämäläinen, H., et al. (2020). Bilingualism is associated with a delayed onset of dementia but not with a lower risk of developing it: a systematic review with metaanalyses. Neuropsychol. Rev. 30, 1-24. doi: 10.1007/s11065-020-09426-8

Burke, S. L., Naseh, M., Rodriguez, M. J., Burgess, A., and Loewenstein, D. (2019). Dementia-related neuropsychological testing considerations in nonHispanic White and Latino/Hispanic populations. Psychol. Neurosci. 12, 144-168. doi: 10.1037/pne0000163

Calabria, M., Hernández, M., Cattaneo, G., Suades, A., Serra, M., Juncadella, M., et al. (2020). Active bilingualism delays the onset of mild cognitive impairment. Neuropsychologia 146:107528. doi: 10.1016/j.neuropsychologia.2020.107528

Calvo, N., García, A. M., Manoiloff, L., and Ibáñez, A. (2016). Bilingualism and cognitive reserve: a critical overview and a plea for methodological innovations. Front. Aging Neurosci. 7:249. doi: 10.3389/fnagi.2015.00249

Chertkow, H., Whitehead, V., Phillips, N., Wolfson, C., Atherton, J., and Bergman, H. (2010). Multilingualism (but not always bilingualism) delays the onset of Alzheimer disease: evidence from a bilingual Community. Alzheimer Dis. Assoc. Disord. 24, 118-125. doi: 10.1097/WAD.0b013e3181ca1221

Craik, F. I., Bialystok, E., and Freedman, M. (2010). Delaying the onset of Alzheimer disease: bilingualism as a form of cognitive reserve. Neurology 75, 1726-1729. doi: 10.1212/WNL.0b013e3181fc2a1c

Cronbach, L. J. (1975). Beyond the two disciplines of scientific psychology. Amer. Psychol. 30, 116-127. doi: 10.1037/h0076829

Estanga, A., Ecay-Torres, M., Ibañez, A., Izagirre, A., Villanua, J., Garcia-Sebastian, M., et al. (2017). Beneficial effect of bilingualism on Alzheimer's disease CSF biomarkers and cognition. Neurobiol. Aging 50, 144-151. doi: 10.1016/j.neurobiolaging.2016.10.013

Fairchild, A. J., and MacKinnon, D. P. (2009). A general model for testing mediation and moderation effects. Prev. Sci. 10, 87-99. doi: 10.1007/ s11121-008-0109-6

Fratiglioni, L., Paillard-Borg, S., and Winblad, B. (2004). An active and socially integrated lifestyle in late life might protect against dementia. Lancet Neurol. 3, 343-353. doi: 10.1016/s1474-4422(04)00767-7

Freedman, M., Alladi, S., Chertkow, H., Bialystok, E., Craik, F. I. M., Phillips, N. A., et al. (2014). Delaying onset of dementia: are two languages enough? Behav. Neurol. 2014:808137. doi: 10.1155/2014/808137

Freret, T., Gaudreau, P., Schumann-Bard, P., Billard, J. -M., and Popa-Wagner, A. (2015). Mechanisms underlying the neuroprotective effect of brain reserve against late life depression. J. Neural Transm. 122, S55-S61. doi: 10.1007/ s00702-013-1154-2

Gollan, T. H., Salmon, D. P., Montoya, R. I., and Galasko, D. R. (2011). Degree of bilingualism predicts age of diagnosis of Alzheimer's disease in loweducation but not in highly educated Hispanics. Neuropsychologia 49, 3826-3830. doi: 10.1016/j.neuropsychologia.2011.09.041

Grady, C. L., Luk, G., Craik, F. I. M., and Bialystol, E. (2015). Brain network activity in monolingual and bilingual older adults. Neuropsychologia 66, 170-181. doi: 10.1016/j.neuropsychologia.2014.10.042

Green, D. W. (1998). Mental control of the bilingual lexico-semantic system. Biling. Lang. Congn. 1, 67-81. doi: 10.1017/s1366728998000133

Grundy, J. G., and Anderson, J. A. E. (2017). Commentary: the relationship of bilingualism compared to monolingualism to the risk of cognitive decline or dementia: a systematic review and meta-analysis. Front. Aging Neurosci. 9:344. doi: 10.3389/fnagi.2017.00344

Guzmán-Vélez, E., and Tranel, D. (2015). Does bilingualism contribute to cognitive reserve? Cognitive and neural perspectives. Neuropsychology 29, 139-150. doi: 10.1037/neu0000105

Hack, E. E., Dubin, J. A., Fernandes, M. A., Costa, S. M., and Tyas, S. L. (2019). Multilingualism and dementia risk: longitudinal analysis of the nun Study. J. Alzheimers Dis. 71, 210-212. doi: 10.3233/JAD-181302

Heredia, R. R., and Cieślicka, A. B. (2014). "Bilingual storage: compoundcoordinate and derivates" in Foundations of bilingual memory. eds. R. R. Heredia and J. Altarriba (NY: Springer), 11-40.

Hilchey, M. D., and Klein, R. M. (2011). Are there bilingual advantages on nonlinguistic interference tasks? Implications for the plasticity of executive control processes. Psychon. Bull. Rev. 18, 625-657. doi: 10.3758/s13423-011-0116-7
Iaccarino, L., Sala, A., Caminiti, S., Presotto, L., and Perani, D. (2020). In vivo MRI structural and PET metabolic connectivity study of dopamine pathways in Alzheimer's disease. J. Alzheimers Dis. 75, 1003-1016. doi: 10.3233/ JAD-190954

Jaeger, F. (2008). Categorical data analysis: away from ANOVAs (transformation or not) and towards logit mixed models. J. Mem. Lang. 59, 434-446. doi: 10.1016/j.jml.2007.11.007

Kavé, G., Eyal, N., Shorek, A., and Cohen-Mansfield, H. (2008). Multilingualism and cognitive state in the oldest old. Psychol. Aging 23, 70-78. doi: 10.1037/0882-7974.23.1.70

Kenny, D. A. (2019). Enhancing validity in psychological research. Am. Psychol. 74, 1018-1028. doi: 10.1037/amp0000531

Klein, R. M., Christie, J., and Pakvall, M. (2016). Does multilingualism affect the incidence of Alzheimer's disease?: a worldwide analysis by country. SSM Popul. Health 2, 463-467. doi: 10.1016/j.ssmph.2016.06.002

Klein, D., Mok, K., Chen, J.-K., and Watkins, K. E. (2014). Age of language learning shapes brain structure: a cortical thickness study of bilingual and monolingual individuals. Brain Lang. 131, 20-24. doi: 10.1016/j.bandl.2013.05.014

Klimova, B., Valis, M., and Kucka, K. (2017). Bilingualism as a strategy to delay the osent of Alzheimer's disease. Clin. Interv. Aging 12, 1731-1737. doi: $10.2147 /$ CIA.S145397

Kousaie, S., and Phillips, N. (2012). Conflict monitoring and resolution: are two languages better than one? Evidence from reaction time and event-related brain potentials. Brain Res. 1446, 71-90. doi: 10.1016/j.brainres.2012.01.052

Kousaie, S., and Phillips, N. A. (2017). A behavioural and electrophysiological investigation of the effect of bilingualism on aging and cognitive control. Neuropsychologia 94, 23-35. doi: 10.1016/j.neuropsychologia.2016.11.013

Kraemer, K. R., Enam, T., and McDonough, I. M. (2019). Cognitive reserve moderates older adults' memory errors in an autobiographical reality monitoring task. Psychol. Neurosci. 12, 247-256. doi: 10.1037/pne0000161

Lancaster, B. P. (1999). Defining and interpreting suppressor effects: advantages and limitations. Paper presented at the Annual Meeting of the Southwest Educational Research Association (San Antonio, TX, January 21-23, 1999). [ERIC number: ED426097: Available at: https://files.eric.ed.gov/fulltext/ ED426097.pdf].

Liu, Y. C., Yip, P. K., Fan, Y. M., and Meguro, K. (2012). A potential protective effect in multilingual patients with semantic dementia: two case reports of patients speaking Taiwanese and Japanese. Acta Neurol. Taiwanica 21, 25-30.

Ljungberg, J. K., Hansson, P., Andrés, P., Josefsson, M., and Nilsson, L. -G. (2013). A longitudinal study of memory advantages in bilinguals. PLoS One 8:e73029. doi: 10.1371/journal.pone.0073029

Lorch, R. F., and Myers, J. L. (1990). Regression analyses of repeated measures data in cognitive research. J. Exp. Psychol. Learn. Mem. Cogn. 16, 149-157. doi: 10.1037//0278-7393.16.1.149

Luk, G., Bialystok, E., Craik, F. I., and Grady, C. L. (2011). Lifelong bilingualism maintains white matter integrity in older adults. J. Neurosci. 31, 16808-16813. doi: 10.1523/jneurosci.4563-11.2011

Martin, J. D., Shipstead, Z., Harrison, T., Redick, T. S., Bunting, M., and Engle, R. W. (2020). The role of maintenance and disengagement in predicting reading comprehension and vocabulary learning. J. Exp. Psychol. Learn. Mem. Cogn. 46, 140-154. doi: 10.1037/xlm0000705

Mondini, S., Guarino, R., Jarema, G., Kehayia, E., Nair, V., Nucci, M., et al. (2014). Cognitive reserve in a cross-cultural population: the case of Italian emigrants in Montreal. Aging Clin. Exp. Res. 26, 655-659. doi: 10.1007/ s40520-014-0224-0

Morales, J., Yudes, C., Gómez-Ariza, C. J., and Bajo, M. T. (2015). Bilingualism modulates dual mechanisms of cognitive control: evidence from ERPs. Neuropsychologia 66, 157-169. doi: 10.1016/j.neuropsychologia.2014.11.014

Mukadam, N., Jichi, F., Green, D., and Livingston, G. (2017a). The relationship of bilingualism to cognitive decline: the Australian longitudinal study of ageing. Int. J. Geriatr. Psychiatry 33, e249-e256. doi: 10.1002/gps.4778

Mukadam, N., Sommerlad, A., and Livingston, G. (2017b). The relationship of bilingualism compared to monolingualism to the risk of cognitive decline or dementia: a systematic review and meta-analysis. J. Alzheimers Dis. 58, 45-54. doi: 10.3233/JAD-170131

Nithianantharajah, J., and Hannan, A. J. (2011). Mechanisms mediating brain and cognitive reserve: experience-dependent neuroprotection and functional compensation in animal models of neurodegenerative diseases. Prog. NeuroPsychopharmacol. Biol. Psychiatry 35, 331-339. doi: 10.1016/j.pnpbp.2010.10.026 
Paap, K. R. (2018). "Bilingualism and executive functioning” in An introduction to bilingualism. 2nd Edn. eds. J. Altarriba and R. R. Heredia (New York: Routledge), 190-224.

Paap, K. R., Johnson, H. A., and Sawi, O. (2014). Are bilingual advantages dependent upon specific tasks or specific bilingual experiences? J. Cogn. Psychol. 26, 615-639. doi: 10.1080/20445911.2014.944914

Papageorgiou, A., Bright, P., Tomas, E. P., and Filippi, R. (2019). Evidence against a cognitive advantage in the older bilingual population. Q. J. Exp. Psychol. 72, 1354-1363. doi: 10.1177/1747021818796475

Paulavicius, A. M., Mizzaci, C. C., Tavares, D. R., Rocha, A. P., Civile, V. T., Schultz, R. R., et al. (2020). Bilingualism for delaying the onset of Alzheimer's disease: a systematic review and meta-analysis. Eur. Geriatr. Med. 11, 651-658. doi: 10.1007/s41999-020-00326-x

Perani, D., and Abutalebi, J. (2015). Bilingualism, dementia, cognitive and neural reserve. Curr. Opin. Neurol. 28, 618-625. doi: 10.1097/wco.0000000000000267

Perani, D., Farsad, M., Ballarini, T., Lubian, F., Malpetti, M., Fraccheti, A., et al. (2017). The impact of bilingualism on brain reserve and metabolic connectivity in Alzheimer's dementia. Proc. Natl. Acad. Sci. U. S. A. 114, 1690-1695. doi: 10.1073/pnas.1610909114

Perquin, M., Vaillant, M., Schuller, A. -M., Pastore, J., Dartigues, J. -F., Lair, M. -L., et al. (2013). Lifelong exposure to multilingualism: new evidence to support cognitive reserve hypothesis. PLoS One 8:e62030. doi: 10.1371/journal. pone. 0062030

Petrocelli, J. V., Clarkson, J. J., Whitmire, M. B., and Moon, P. E. (2013). When $\mathrm{ab} \neq \mathrm{c}-\mathrm{c}^{\prime}$ : published errors in the reports of single-mediator models. Behav. Res. Ther. 45, 595-601. doi: 10.3758/s13428-012-0262-5

Preacher, K. J., and Hayes, A. F. (2008). Asymptotic and resampling strategies for assessing and comparing indirect effects in multiple mediator models. Behav. Res. Methods 40, 879-891. doi: 10.3758/BRM.40.3.879

Reyes, A., Paul, B. M., Marshall, A., Chang, Y. -H. A., Bahrami, N., Kansal, L., et al. (2018). Does bilingualism increase brain or cognitive reserve in patients with temporal lobe epilepsy? Epilepsia 59, 1037-1047. doi: 10.1111/ epi. 14072

Rossi, E., Cheng, H., Kroll, J. F., Diaz, M. T., and Newman, S. D. (2017). Changes in white-matter connectivity in late second language learners: evidence from diffusion tensor imaging. Front. Psychol. 8:2040. doi: 10.3389/ fpsyg.2017.02040

Sauter, J., Widmer, E., Ihle, A., and Kliegel, M. (2019). The association of leisure activities in middle adulthood with cognitive performance in old age: social capital mediates cognitive reserve effects. Psychol. Neurosci. 12, 236-246. doi: 10.1037/pne0000146

Scarmeas, N., Albert, S. M., Manly, J. J., and Stern, Y. (2006). Education and rates of cognitive decline in incident Alzheimer's disease. J. Neurol. Neurosurg. Psychiatry 77, 308-316. doi: 10.1136/jnnp.2005.072306

Scarmeas, N., Levy, G., Tang, M.-X., Manly, J., and Stern, Y. (2001). Influence of leisure activity on the incidence of Alzheimer's disease. Neurology 57, 2236-2242. doi: 10.1212/wnl.57.12.2236

Schweizer, T. A., Ware, J., Fischer, C. E., Craik, F. I., and Bialystok, E. (2012). Bilingualism as a contributor to cognitive reserve: evidence from brain atrophy in Alzheimer's disease. Cortex 48, 991-996. doi: 10.1016/j. cortex.2011.04.009
Simonton, D. K. (2008). "Bilingualism and creativity" in An introduction to bilingualism: Principles and processes. eds. J. Altarriba and R. R. Heredia (NY: Lawrence Erlbaum Associates), 147-166.

Stern, Y., Albert, S., Tang, M. -X., and Tsai, W. -Y. (1999). Rate of memory decline in $\mathrm{AD}$ is related to education and occupation. Cognitive reserve? Neurology 53, 1942-1947. doi: 10.1212/wnl.53.9.1942

Stern, Y., Gurland, B., Tatemichi, T. K., Tang, M. X., Wilder, D., and Mayeux, R. (1994). Influence of education and occupation on the incidence of Alzheimer's disease. JAMA 271, 1004-1010. doi: 10.1001/jama.1994.03510370056032

Stern, Y., Tang, M. X., Denaro, J., and Mayeux, R. (1995). Increased risk of mortality in Alzheimer's disease patients with more advanced educational and occupational attainment. Ann. Neurol. 37, 590-595. doi: 10.1002/ana.410370508

Thorvaldsson, V., Skoog, I., and Johansson, B. (2017). IQ as moderator of terminal decline in perceptual and motor speed, spatial, and verbal ability: testing the cognitive reserve hypothesis in a population-based sample followed from age 70 until death. Psychol. Aging 32, 148-157. doi: 10.1037/pag0000150

Valenzuela, M. J., and Sachdev, P. (2006a). Brain reserve and cognitive decline: a non-parametric systematic review. Psychol. Med. 36, 1065-1073. doi: 10.1017/ s0033291706007744

Valenzuela, M. J., and Sachdev, P. (2006b). Brain reserve and dementia: a systematic review. Psychol. Med. 36, 441-454. doi: 10.1017/s0033291705006264

Van Dijk, K. R. A., Van Gerven, P. W. M., Van Boxtel, M. P. J., Van der Elst, W., and Jolles, J. (2008). No protective effects of education during normal cognitive aging: results from the 6-year follow-up of the Maastricht aging study. Psychol. Aging 23, 119-130. doi: 10.1037/0882-7974.23.1.119

Woumans, E. V. Y., Santens, P., Sieben, A., Versijpt, J. A. N., Stevens, M., and Duyck, W. (2015). Bilingualism delays clinical manifestation of Alzheimer's disease. Biling. Lang. Congn. 18, 568-574. doi: 10.1017/S136672891400087X

Woumans, E., Versijpt, J., Sieben, A., Santens, P., and Duyck, W. (2017). Bilingualism and cogntive decline: a story of pride and prejudice. J. Alzheimers Dis. 60, 1237-1239. doi: 10.3233/JAD-170759

Xie, Z., and Zhou, S. (2020). Bilingualism, demographics, and cognitive control: a within-group approach. Front. Psychol. 11:94. doi: 10.3389/fpsyg.2020.00094

Yzerbyt, V., Muller, D., Batailler, C., and Judd, C. M. (2018). New recommendations for testing indirect effects in mediational models: the need to report and test component paths. J. Pers. Soc. Psychol. 115, 929-943. doi: 10.1037/pspa0000132

Zahodne, L. B., Schofield, P. W., Farrell, M. T., Stern, Y., and Manly, J. J. (2014). Bilingualism does not alter cognitive decline or dementia risk among Spanish-speaking immigrants. Neuropsychology 28, 238-246. doi: 10.1037/ neu0000014

Conflict of Interest: The authors declare that the research was conducted in the absence of any commercial or financial relationships that could be construed as a potential conflict of interest.

Copyright (C) 2020 Heredia, Blackburn and Vega. This is an open-access article distributed under the terms of the Creative Commons Attribution License (CC BY). The use, distribution or reproduction in other forums is permitted, provided the original author(s) and the copyright owner(s) are credited and that the original publication in this journal is cited, in accordance with accepted academic practice. No use, distribution or reproduction is permitted which does not comply with these terms. 